\title{
Uncovering Complexity in the Jakarta Energy Planning Process using Agent-Oriented Analysis
}

\section{Yohanes Dwi Anggoro}

School of Systems, Management and Leadership

University of Technology Sydney

New South Wales, Australia

Email: yohanes.d.anggoro@student.uts.edu.au

\section{Suwin Sandu}

School of Systems, Management and Leadership

University of Technology Sydney

New South Wales, Australia

Email: suwin.sandu@uts.edu.au

\section{Ghassan Beydoun}

School of Systems, Management and Leadership

University of Technology Sydney

New South Wales, Australia

Email: ghassan.beydoun@uts.edu.au

\begin{abstract}
The Jakarta Energy Planning Process (JEPP) is expected to be a successful template for other provinces in Indonesia. However, JEPP consists of a complex set of interrelated activities. These activities are fraught with difficulties and errors, including incorrectness, inconsistency, incompleteness, and redundancy in the process under which the Jakarta energy planning is undertaken. This paper aims to identify complexity issues in JEPP with the aim to alleviate these complexities using Agent-Oriented Analysis (AOA). This research uses the Design Science Research (DSR) method and towards the analysis employs seven Agent-Based Modellings (ABMs), including goal model, role model, organisation model, interaction model, environment model, agent model, and scenario model. The research consists of five stages: the synthesis of a preliminary knowledge analysis framework, the identification of complexity issues, recommendation synthesis, and finally the development of the complete knowledge analysis framework. While the analysis undertaken in this paper focuses on Jakarta, the developed knowledge analysis framework should be useful for energy planners in other regions, and research communities in general who are involved in such endeavours in developing complex planning processes.
\end{abstract}

Keywords: Agent Oriented Analysis, Energy Planning Process, Knowledge Framework, Complexity Issues.

\section{Introduction}

Jakarta, as the capital city of Indonesia, is expected to act as a role model for the achievement of the National Energy Policy targets set by the National Energy Council. All team members and energy stakeholders involved in the Jakarta Energy Planning Team are also expected to plan future energy needs effectively and to contribute significantly to meet the national energy mix in a sustainable and an environmentally friendly manner. However, energy planning activities and processes are inherently complex. Many inconsistency problems and constraint violations invariably arise in system development and implementation process, in particular where knowledge modelling is incrementally undertaken (Sarmiento et al. 2015; Beydoun et al. 1998). According to Balint et al (2011), some of the common problems that may arise in the energy planning system such as a lack of a single problem statement, administrative and scientific complexity, conflicting data values, conflicting objectives, political complexity, a dynamic and a changing context, and multiplicity of actors involved. Furthermore, another common problem for a process system is the duplication of efforts in the process. Repetitive steps often reduce the quality of the process and confuse the actors involved. Collecting data and maintaining its quality are also major challenges in the energy planning process both nationally and regionally (Cajot et al. 2017). It is paramount to clearly identify the core issues in the Jakarta energy planning process to formulate the complex energy planning process and this is yet to be adequately undertaken (Cajot et al. 2017). 
The objectives of this paper is to improve JEPP. The paper provides a preliminary knowledge analysis framework aiming to provide a recommendation to reduce complexity issues in the energy planning process. The main research question in this paper is "How can Agent-Oriented Analysis (AOA) alleviate several challenges of energy planning process?”. Towards a problem solving through effective and efficient analysis of information system, this research deploys seven ABMs from the Agent-Oriented Software Engineering (AOSE) practice. The models have proved sufficient to cover more than 20 AOSE existing methodologies (Beydoun et al. 2009; Lopez-Lorca et al. 2016). This approach has proved effective in identifying and developing several issues in a complex system (Liang et al. 2013; Miller et al. 2014; Shvartsman and Taveter 2014; Inan 2015). The chosen ABMs are goal model, role model, organisation model, interaction model, environment model, agent model, and scenario model. The use of this methodology addresses a broader research complexity management question by constructing and evaluating knowledge artefact design to understand and solve human and organization complex problems.

The rest of this paper is organized as follows: The next section reviews the development of agent technology and ABMs in the area of complex systems. The third section describes the methodology used in this research. The fourth section presents the overview of JEPP. The fifth section provides the research results and discussions on knowledge analysis framework. Finally, the sixth section concludes with a discussion of future work.

\section{Related Work}

The use of the term agent has been widely used, including in the field of informatics and computer science, industrial, manufacturing, business, and electronic commerce. It resulted in the increasingly unclear definition of agent, as any researcher trying to define the agent with the background knowledge they have (Wooldridge \& Jennings 1995). In the development of agent technology, there are more numerous and complicated tasks that have to be done by an agent in a system, and it requires more agents in a system to complete those tasks. The paradigm of system development where there are multiple agents in a system, which interacts, negotiates and coordinate each other to carry out and complete the tasks is called as the Multi-Agent System (MAS). The MAS as sub-field of the Artificial Intelligence (AI) aims to provide construction principles of a complex system involving several agents and coordination mechanisms for behaviours of the independent agents. Therefore, the MAS approach also can fulfil the user needs and meet the user's requirements, which can be very complex (Al-azawi and Ayesh 2013), for instance peer-to-peer community based searching systems (Beydoun et al. 2011), and supply chain management (Xu et al. 2011).

The system development is often interpreted as a process to improve existing system by developing a new system to replace the old system. There are some reasons the old system needs to be repaired or replaced, such as the existence of various problems, irregularities in the system, the growth of the organization, to achieve a greater chance, the system changes requested by the user. According to Akbari (2010), AOSE methodology is a business process of system development by using different concept and modeling tools of an agent, and put agents as central modelling to evolve system development paradigm (Wooldridge 1999; Ashamalla et al. 2017). It has been widely used in many domains, including robotics, networking, security, traffic control, gaming and commerce (Akbari 2010), disaster management (Inan et al. 2015). Furthermore, Lopez-Lorca (2016) argue that the Agent-Oriented Analysis (AOA) as an analysis phase in a development life cycle based on AOSE methodology, which aims to capture some knowledge characteristics of existing system that need to be developed. Some literatures have demonstrated the effectiveness of AOA to capture complex knowledge characteristics of a particular domain, which contain various activities and constraints. For example, the use of AOA for the system development of health decision support framework (Liang et al. 2013), military training scenarios (Shvartsman and Taveter 2014), and aircraft turnaround simulation (Miller et al. 2014).

\section{Research Methodology}

The selection of methodology in this research is vital as it will directly affect the achievement of the study objectives and results (Silver et al. 1995, Zmud 1997). This research use the Design Science Research (DSR) method to assist a problem solving through effective and efficient analysis of information system (Hevner et al., 2004). The purpose of employing the DSR method in this research is to develop a knowledge analysis framework for identifying real complex problem in energy planning processes and providing recommendation to improve these processes. 


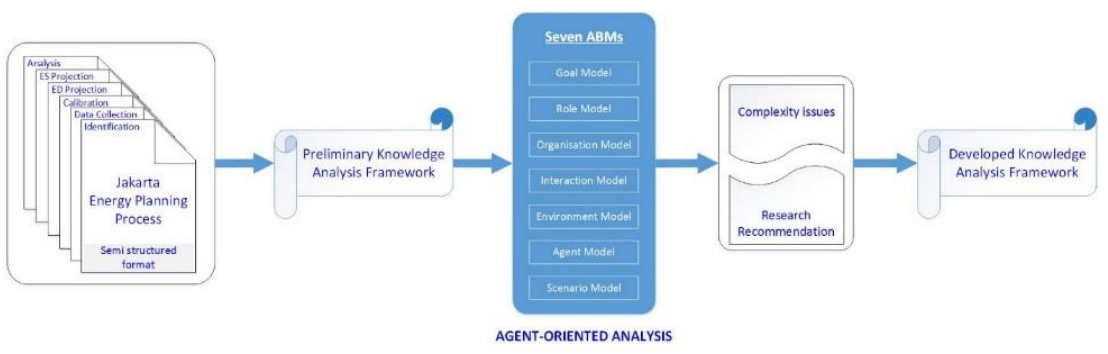

Figure 1. The Agent-Oriented Analysis (AOA) Stages.

Figure 1 describes several stages in this research. Firstly, the input of this research comes from JEPP workflow, which contains detail information of the energy planning process. Secondly, the preliminary knowledge analysis framework is developed based on information contained in JEPP workflow. Thirdly, the AOA of the preliminary knowledge analysis framework is undertaken using seven ABMs in the AOSE methodology to synthesise and to capture complex knowledge of JEPP. Fourthly, based on AOA results, the real complexity issues of JEPP is identified. Finally, the output of this research will provide developed knowledge analysis framework by considering the research recommendation results.

\section{Jakarta's Energy Planning Process}

Generally, the energy planning process at either regional or national level requires a diversity of inputs and coordination of various fields, stakeholders and information to achieve a goal of developing sustainable energy systems. The JEPP consists of six main steps, including energy issues and study goal identification, overview of existing energy supply system, calibration of energy system modelling, energy demand projection, energy supply projection, and results analysis. According to the Jakarta Governor Decree No. 989 Year 2017, the study team of the Regional Energy General Plan (REGP) of the Jakarta province consists of six main organizational elements, including University (U), Districts (D), Regional Planning Agency (RPA), Regional Secretariat (RS), Regional Department (RD), and Regional Technical Institution (RTI). Each of these organisations has several actors who involve directly on the study implementation and responsible to the study coordinator. In total, there are more than 20 actors as team members. In addition, it also involves some energy stakeholders in 10 organisations at national level to support the study team in collecting socio-technical data to complete the regional energy planning study.

\section{Results and Discussion}

\subsection{Preliminary Knowledge Analysis Framework}

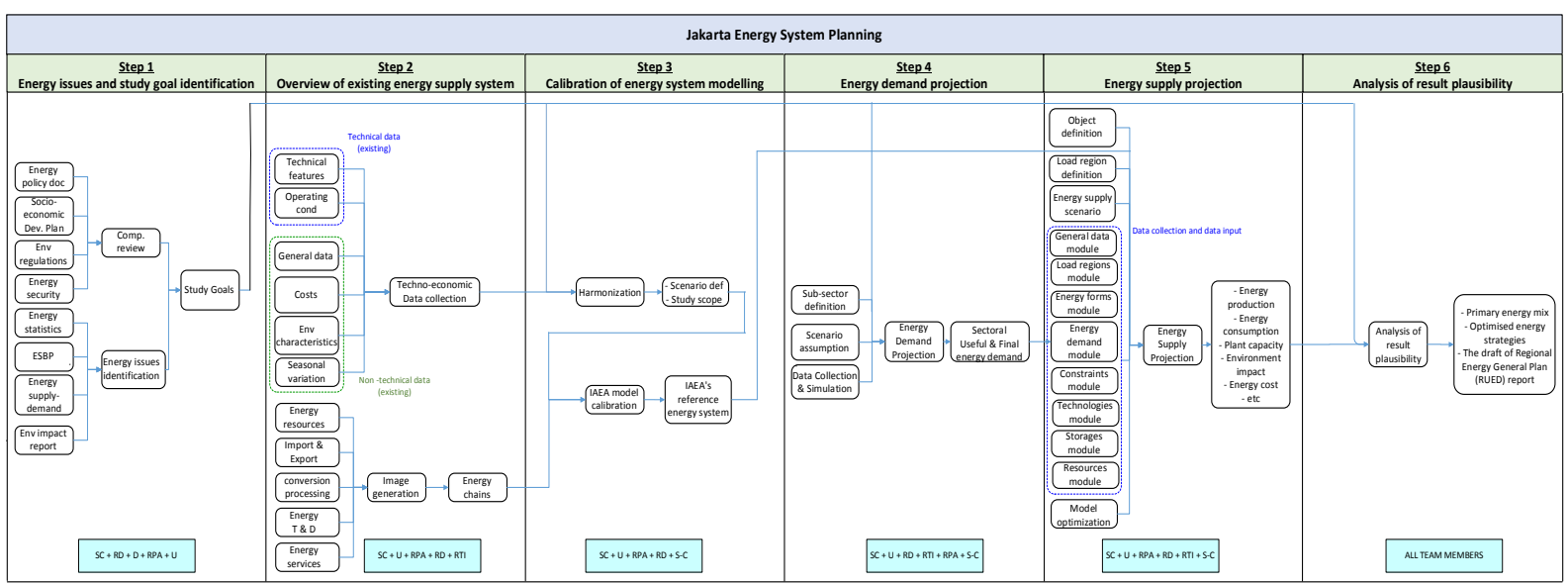

Figure 2. Preliminary knowledge analysis framework

Figure 2 presents the preliminary knowledge analysis framework of JEPP, which shows all activities and actors involved in each process. This preliminary framework aims to capture all existing knowledge of JEPP in a comprehensive and systematic manner based on information described in previous section, so that the complex energy planning process can be relatively easily understood. As a result, the preliminary knowledge analysis framework of JEPP as shown in Figure 2 able to describe all detail energy planning activities performed by the Jakarta energy planning team sequentially and systematically. 


\subsection{Agent-Oriented Analysis}

This section describes the agent-oriented analysis within the preliminary knowledge analysis framework. It details the generation of several ABMs including goal model, role model, organisation model, interaction model, environment model, agent model, and scenario model.

\subsubsection{The goal model}

This research starts with the goal model at the beginning of the AOA, and put it as central modelling because it will become the basis for other ABMs to be processed. This research identified all six main goal models and 17 sub-goal models, which cover more than 90 activities or steps of the Jakarta energy planning process. Furthermore, it also identified each role involved in each goal and sub-goal. These goal models are identified to describe and explain the purpose and goal hierarchy in JEPP. It also presents some knowledge elements, including: First, there are two knowledge elements, i.e. the goal (a main goal and 17 sub-goals) and the role. Second, the objective of energy planning activities is to achieve the main goal. Third, the main goal may consists of several sub-goals that must be responsible to achieve the main goal by having element "how-to" achieve the main goal. Fourth, the role(s) refer to the agent role to chase the sub-goal(s).

\subsubsection{The role model}

The role model can be processed after the goal model as the role model represents more detail on responsibilities and constraints that has been identified in the goal model to achieve the system's goal. This research identified 36 role models, which explain responsibilities of 36 actors/agents involved in the energy planning process based on the Governor Decree No. 989/2017 and real events. The role model presents some knowledge elements, including: First, the unique role ID that obtained from the goal model. Second, the role name element that describes the agent's role. Third, the description element that provides an explanation about the agent's role. Fourth, the responsibilities element that describes all roles in order to achieve the goal or sub-goals models. Fifth, the constraints element that defines the condition of the role's entities (organisations/agencies/individuals).

\subsubsection{The organisation model}

The organisation model is performed based on hierarchy level of the agent involved in the goal model and the role model. This research identified several organization models for 36 agents involved in JEPP. It aims to inform the work relationship, coordination, communication and negotiation between those agents. It presents two knowledge element namely: the roles that obtained from the goal models and the relationship that describe how the roles are related, coordinated, communicated and negotiated each other. Generally, there are two relationship types i.e. isControlledBy relationship, which define that one of the interrelated roles is in higher administration level and have an authority to control others; and isPeer relationship, which define that both of the interrelated roles has same level position and does not have an authority to control each other.

\subsubsection{The interaction model}

The interaction model is performed to connect several activities with their agent's roles, in which each connection represent an activity that has to be done to achieve main goal or sub-goal defined in the goal model. The knowledge elements captured in the interaction models are as follows: First, the role element is identified based on the goal model and the role model. Second, the activity element, which represent some activities in the sub-goals to achieve the main goal identified in the goal model. Third, the interaction element, which describe the relationship between activities and the agent's roles.

\subsubsection{The environment model}

The aim of the environment model is to identify environment entity that used by MAS when they pursue main goal or sub-goals defined in the goal model. This research identified twelve environment models where each environment entity also has some environment attributes. Furthermore, the environment model presents some knowledge elements: First, the unique environment entity ID for each environment element. Second, the environment name element that describes the environment type. Third, the description element that provides an explanation about the environment entity. Fourth, the attribute element that provide environment attribute list. Fifth, the role element that inform several agent's roles who use this environment model. 


\subsubsection{The agent model}

The agent model is required to inform a set of equipment owned by one particular agent or actor to achieve each main goal or sub-goal defined in the goal model. This research identified 27 agent models, which explain more detail about all activities trigger and action, and environment entities of each actor. There are many knowledge element captured in the agent model, including agent name element, description element, reference element that obtained from the role model, environment element that obtained from the environment model, and activity element that provide activities list performed by agent to achieve its main goal or sub goal. Then, in each activity element has sub-element such as activity name element, functionality element that describes which main goal or sub-goals need to be achieved in the activity, trigger element that indicates what event will cause the activity start, and action element that describe a set of activities need to be performed when the activity started.

\subsubsection{The scenario model}

The scenario model as the last of AOA is performed to provide detail descriptions of event sequences to achieve main goal or sub-goals defined in the goal model. This research created 17 scenario models, which describe how each scenario starts and ends. It also has many knowledge elements that similar with the knowledge elements in the agent model. However, the activity element in the scenario model provide all activities list, in which set up together with the role element, and the environment element required to achieve particular objective. Then, the condition element aims to inform whether those activities performed in parallel, sequentially or interleaved way. So that, this scenario model can be understood comprehensively and more easily.

\subsection{Complexity Issues of the Jakarta Energy Planning Process}

Based on the results of AOA using the ABMs in the previous section, this research identifies several complex problems in the Jakarta energy planning process, as shown in Figure 3. It shows that there are four categories of complex problems: Firstly, incorrectness may occur when the requirements obtained do not accurately reflect the client's future needs. Secondly, inconsistency may occur when two or more users have conflicting requirements. Thirdly, incompleteness may occur when the clients are not fully understand the overall impact of the current decision. Fourthly, redundancy usually occurs because of the lack of collaboration between departments, so a process has been adapted in a less systematic way.

In case of JEPP, this research identified complexity issues occurred in JEPP, such as: the redundancy issues (repetition task) and the inconsistency issues (conflicting values) occurred in data collection process in step 2, 4, and 5 of JEPP; and data harmonization process between step 3, and step 2, 4, 5 of JEPP. The incorrectness issues (scientific complexity and uncertainty) and the inconsistency issues (lack of a single problem statement) occurred in energy issues identification process in step 1 of JEPP. Further details, this research identified the redundancy issues of data collection process when there are several data collected in step 2, 4, and 5 are similar. For example, step 2 collects several data of technical features data, operating condition data, general data (study timeframe, discount rate), energy cost data, environmental characteristics (emission, waste, land use), and seasonal variation data (load region, load curve). In the same way, the data collection process in step 4 and 5 collect these data obtained in step 2.

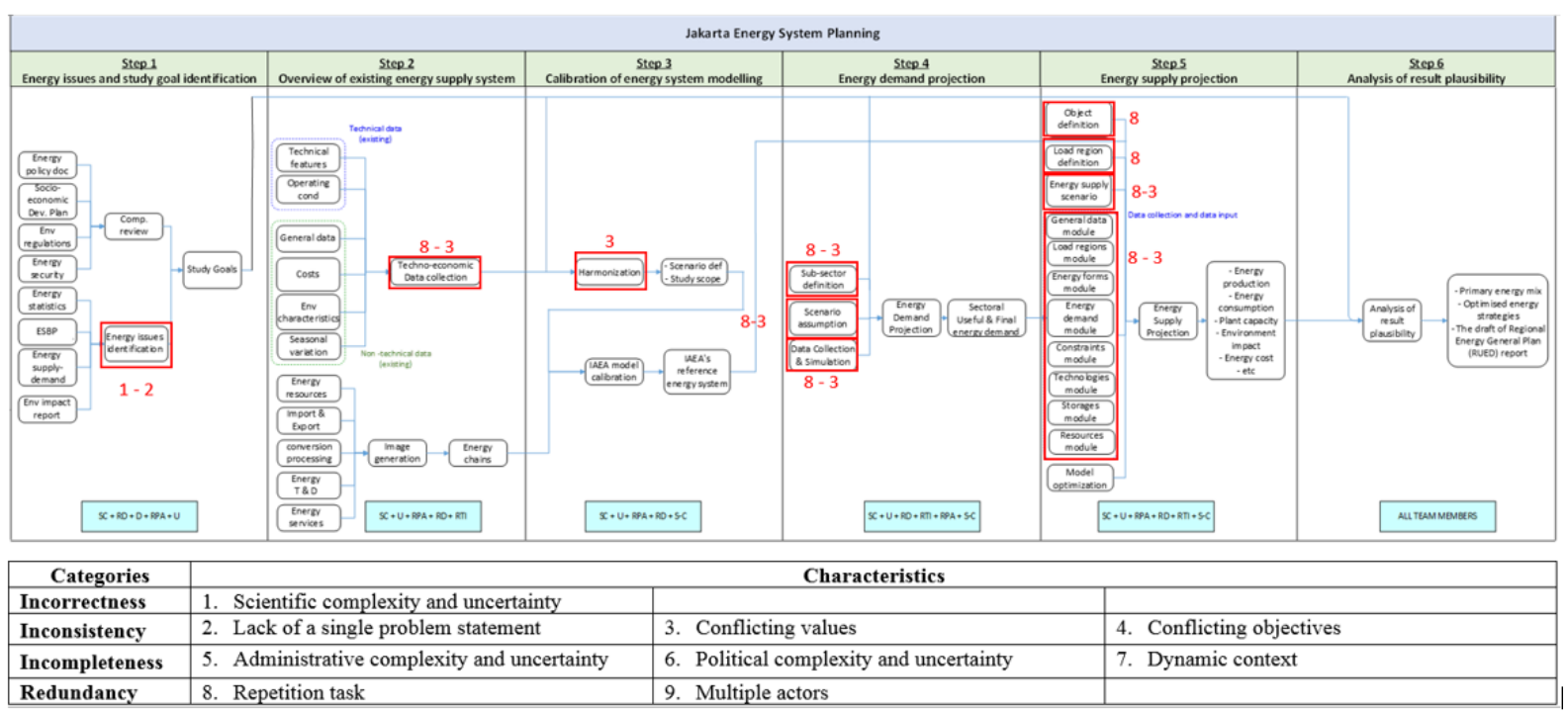

Figure 3. Several complex problem of the Jakarta energy planning process. 


\subsection{Developed Knowledge Analysis Framework}

We provide a number of recommendations to minimize the complexity and to obtain a more efficient process by providing the developed knowledge analysis framework of JEPP as shown in Figure 4. This provides a significant improvement in the energy planning process. The most significant improvement is in the data collection process, conducted iteratively in steps 2,4 , and 5 . This research simplifies the data collection process by consolidating this process in step 2 (techno-economic data collection activity) and removing the process in steps 4 and 5. Secondly, the process of sub-sector definition and scenario assumption for energy demand and energy supply system in steps 4 and 5 are simplified by merging it with the process of data harmonisation in step 3. Thirdly, this research simplifies the process of energy chain generation in step 2 by redefining the required aspects in the energy chain system to become more efficient. By reducing some complexities and providing significance improvements in the energy planning process, it is expected that the developed knowledge analysis framework in this research can be used and adopted as a guidance for energy practitioner, energy researcher, academics and energy stakeholders to perform energy planning process in a more efficient way.

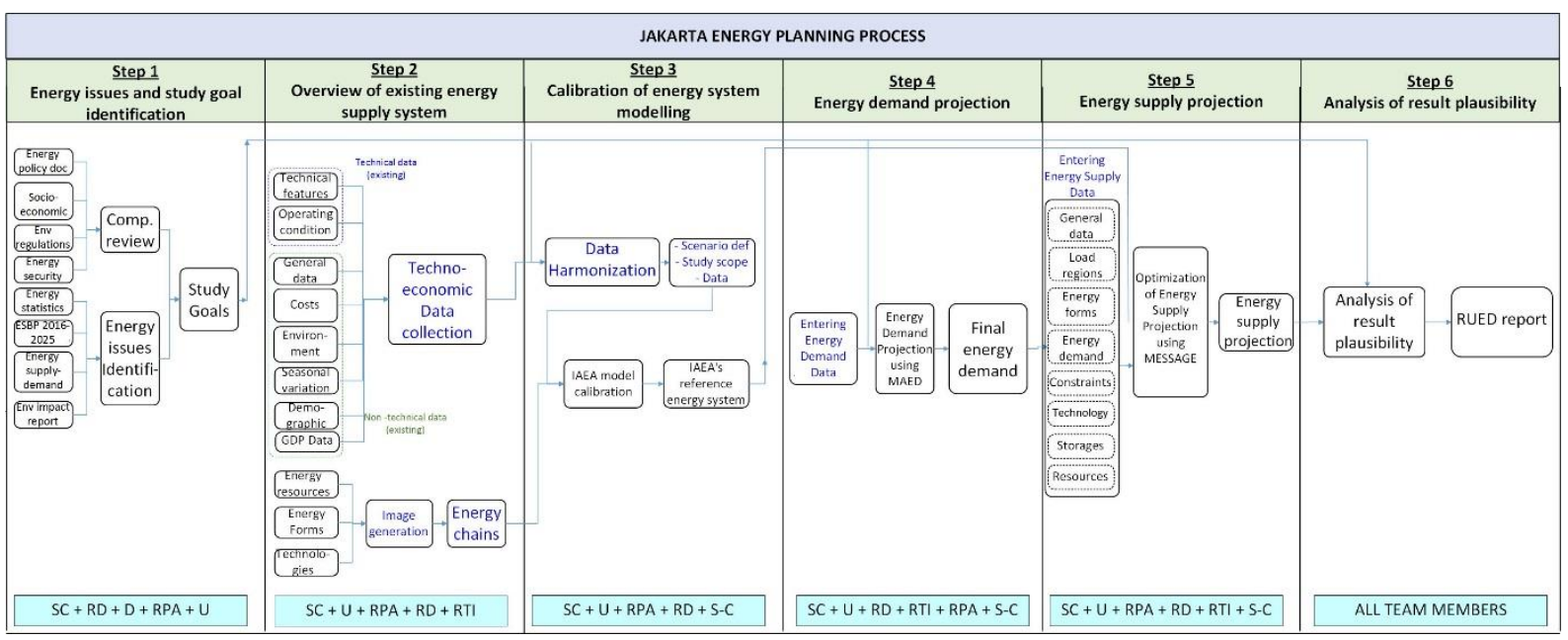

Figure 4. The developed knowledge analysis framework.

\section{Summary and Future Research}

The key results of this research are as follows: Firstly, this research has identified the preliminary knowledge analysis framework based on several information obtained from JEPP. Secondly, this research has captured, and analysed all knowledge artefact of the JEPP by using seven ABMs including goal model, role model, organisation model, interaction model, environment model, agent model, and scenario model. The use of ABMs has helped in understanding the complexity of JEPP in a comprehensive and systematic manner. Thirdly, this research has identified some complex problems in the JEPP. Fourthly, this research has provided several recommendations to reduce complex problems in the Jakarta energy planning process. Finally, this research has developed the knowledge analysis framework based on the result of AOA. For further development, following the DSR method, the next stage of this research will develop more in-depth understanding into the developed knowledge analysis framework obtained in this research. This will enhance the generalisability of the proposed framework to apply it in other countries or regions that similarly have complex planning processes.

\section{References}

Akbari, Z. 2010. "A Survey of Agent-oriented Software Engineering Paradigm: Towards Its Industrial Acceptance," Journal of Computer Engineering Research (1), pp 14-28.

Ashamalla, A., Beydoun, G., and Low, G. 2017. "Model driven approach for realtime requirement analysis of multi-agent systems," Computer Languages, Systems \& Structures (50), pp 127-139.

Al-Azawi, R., and Ayesh, A. 2013. "Comparing Agent -Oriented Programming Versus Object-Oriented Programming," ICIT 2013 The 6th International Conference on Information Technology, pp 2429, Jordan, 8-10 May 2013, IEEE Jordan Chapter.

Balint, P.J., Stewart, R.E., Desai, A. and Walters, L.C. 2011. Wicked Environmental Problems: Managing uncertainty and conflict. Island Press. 
Beydoun, G., Low, G., Henderson-Sellers, B., Mouratidis,H., Gomez-Sanz, J.J., Pavón, J., and GonzalezPerez, C. 2009. "FAML: a generic metamodel for MAS development", IEEE Trans.Softw.En (99: 1), pp 841-863.

Beydoun, G., Low, G., Tran, N., and Bogg, P. 2011. "Development of a peer-to-peer information sharing system using ontologies," Expert Systems with Applications (38:8), pp 9352-9364.

Beydoun, G., Hoffmann, A. 1998. "Simultaneous modelling and knowledge acquisition using NRDR",

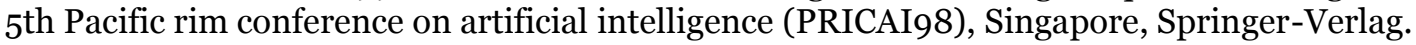

Cajot, S., Peter, M., Bahu, J.M., Guignet, F., Koch, A., and Maréchal, F. 2017. "Obstacles in Energy Planning at the Urban Scale," Sustainable Cities and Society (3o), pp 223-236.

Hevner, A.R., March, S.T., Park, J., and Ram, S. 2004. “Design science in information system research,” MIS Quarterly (28:1), pp 75-105.

Inan, D. I., Beydoun, G., and Opper, S. 2015. "Towards Knowledge Sharing in Disaster Management: an Agent-Oriented Knowledge Analysis Framework," in Proceedings of the 26th Australasian Conference on Information Systems (ACIS2015), Adelaide, South Australia, pp 1-13.

Jennings, N.R., and Wooldridge, M.J. 2001. Agent-Oriented Software Engineering: Handbook of Agent Technology. AAAI/MIT Press.

Lopez-Lorca, A. A., Beydoun, G., Valencia-Garcia, R., and Martinez-Bejar, R. 2016. "Supporting AgentOriented Requirement Analysis with Ontologies," International Journal of Human-Computer Studies (87), pp 20-37.

Liang, Xiao, Fox, J., and Hong, Zhu. 2013. "An Agent-Oriented Approach to Support Multidisciplinary Care Decisions," Paper presented at the 3rd Eastern European Regional Conference on the Engineering of Computer Based Systems (ECBS-EERC).

Miller, T., Lu, B., Sterling, L., Beydoun, G., and Taveter, K. 2014. "Requirements Elicitation and Specification Using the Agent Paradigm: The Case Study of an Aircraft Turnaround Simulator," IEEE Transactions on Software Engineering 40(10), pp 1007-1014.

Sarmiento, E., Leite, J.C.S.d.P., and Almentero, E. 2015. "Using correctness, consistency, and completeness patterns for automated scenarios verification," 2015 IEEE Fifth International Workshop on Requirements Patterns (RePa).

Shvartsman, I., and Taveter, K. 2014. "From Agent-Oriented Models to Profile Driven Military Training Scenarios" in Zavoral, Filip, Jung, Jason, J., and Badica, Costin (Eds.), Intelligent Distributed Computing VII (511), pp 317-322, Springer International Publishing.

Silver, M.S., Markus, M.L., and Beath, C.M. 1995. "The Information Technology Interaction Model: A Foundation for the MBA Core Course," MIS Quarterly (19:3), pp 361-390.

Wooldridge, M., and Jennings, N,R. 1995. "Intelligent agents: Theory and Practice," Knowledge Engineering Review (10:2).

Wooldridge, M., Jennings, N.R., and Kinny, D. 1999. "A Methodology For Agent-oriented Analysis \& Design," Proceedings of the $3^{\text {rd }}$ annual conference on Autonomous Agents AGENTS '99, pp 69-76.

Wooldridge, M. 2002. An Introduction to MultiAgent Systems. John Wiley \& Sons, LTD.

Xu, D., Wijesooriya, C., Wang, Y.-G., and Beydoun, G. 2011. "Outbound logistics exception monitoring: A multi-perspective ontologies' approach with intelligent agents," Expert Systems with Applications (38:11), pp 13604-13611.

Zmud, R. 1997. "Editors Comments," MIS Quarterly (21: 2), pp xxi-xxii.

\section{Copyright}

Copyright: (C) 2018 Yohanes Dwi Anggoro, Suwin Sandu, and Ghassan Beydoun. This is an open-access article distributed under the terms of the Creative Commons Attribution-NonCommercial 3.0 Australia License, which permits non-commercial use, distribution, and reproduction in any medium, provided the original author and ACIS are credited. 